Proceedings of the 2012 Winter Simulation Conference

C. Laroque, J. Himmelspach, R. Pasupathy, O. Rose, and A.M. Uhrmacher, eds

\title{
TOWARDS THE SMART CONSTRUCTION SITE: IMPROVING PRODUCTIVITY AND SAFETY OF CONSTRUCTION PROJECTS USING MULTI-AGENT SYSTEMS, REAL-TIME SIMULATION AND AUTOMATED MACHINE CONTROL
}

\author{
Amin Hammad \\ Professor, Concordia University \\ Concordia Institute for Information Systems \\ Engineering \\ 1515 Ste-Catherine Street West \\ H3G 2W1 Montreal, Canada
}

\author{
Faridaddin Vahdatikhaki, Cheng Zhang, \\ Mohammed Mawlana, Ahmad Doriani \\ Graduate Students, Concordia University \\ Department of Building, Civil, and Environmental \\ Engineering \\ 1515 Ste-Catherine Street West \\ H3G 2W1 Montreal, Canada
}

\begin{abstract}
With the increasing complexity of construction projects grows the challenge of securing safety and achieving desirable productivity as the chief priorities of the construction industry. Addressing these issues requires robust mechanisms for on-site real-time data capturing, information processing and decision-making. The current research aims to further investigate the concept of the Smart Construction Site where workers, equipment, and materials are continuously tracked, and the collected information is processed in near real time to update the design model and the simulation of upcoming tasks, and to provide navigation guidance and safety warnings in case of potential collisions. The objective of our research is to improve the productivity and safety of heavy construction projects by integrating $3 \mathrm{D}$ design models (e.g. highway models) with the managerial and operational processes of heavy construction using advanced agent technology and multi-agent systems, real-time simulation, and automated machine control.
\end{abstract}

\section{INTRODUCTION}

Improving productivity and safety of construction projects is among the main priorities of the construction industry (Beavers et al. 2006). New tracking technologies are providing tools for improving productivity and safety by enabling on-site data capturing and decision making using the Global Positioning System (GPS), Real-Time Location Systems (RTLSs), and other geo-positioning technologies (Perkinson, Bayraktar and Ahmed 2010; Teizer et al. 2010). These tracking technologies are integrated with 3D design models and Digital Terrain Models (DTMs) in two modes: (1) Automated Machine Guidance (AMG): which supports the machine operator through provision of continuous guidance on the digital screen mounted in the cabin of the machine, or (2) Automated Machine Control (AMC): where the position, elevation, and orientation of an earthwork machine are measured using GPS and/or other types of sensors, and used to control the blade position of the machine, eliminating the need for staking or other manual measurements. AMC is used mainly in earthwork projects but can be also used in other operations (Peyret et al. 2000).

Previous research has indicated that AMC has the potential to improve the quality and efficiency of construction projects and to enhance the ability of agencies and contractors to deliver projects safer, faster, and cheaper (Dunston and Monty 2009; Frantzen, Kirn and Kaak 2010; JCMMRI 2012; Peyret et al. 2000; Rausch, Schreiber and Diegelmann 2010; Vonderohe 2009). However, there are several challenges that have to be overcome in order to maximize the benefits of using these technologies in the 3D survey- 
ing-design-contract-construction-inspection workflow (Dunston and Monty 2009,Torres and Ruiz 2011, Vonderohe 2009). These challenges can be classified to technical, administrative, and financial.

In our previous research, we have investigated the concept of the Smart Construction Site (Zhang et al. 2009) where workers and equipment are continuously tracked. The collected information is processed in near real time to update the design model and the simulation of upcoming tasks, and to provide navigation guidance and safety warnings. The present research proposes adding intelligent decision-making at different operational and managerial levels. This will help overcome some of the above challenges.

The objectives of this paper are: (1) To review the state-of-practice and state-of-the-art of the usage of AMC and related technologies; (2) To propose a high-level system design of a Multi-Agent System (MAS), integrated with Real-Time Simulation (RTS) to coordinate AMC site operations and resolve operational and managerial conflicts; and (3) To demonstrate the potential benefits of the proposed approach using several hypothetical case studies.

\section{LITERATURE REVIEW}

\subsection{Automated Machine Control}

The advances in GPS technology have induced many researches on the application of the GPS for automation of construction equipment in pursuit of increased productivity, improved safety and reduced costs.

Peyret et al. (2000) developed two separate systems for the real-time control and monitoring of compactors and pavers. Perkinson, Bayraktar and Ahmed (2010) proposed a framework to make use of GPScollected data for enhanced management and decision-making purposes. The central objective of their methodology is to ensure that all productivity-related data can be efficiently collected in the course of project development and further use this data for planning, scheduling and control.

Several systems are under development to provide telematics information of the AMC equipment to and from a central office, job trailer and other equipment, such as Connected Site and SiteLINK 3D (Noland 2010). Similar systems are already available in the surface mining industry (Jamasmie 2010). Machine-to-Machine (M2M) and Machine-to-Office/Office-to-Machine (M2O/O2M) communications allow for sharing different types of data, such as machine condition, location, productivity, work quality, etc.

Some governmental bodies and construction companies have started to appreciate the value of employing AMC in heavy earthmoving and road construction projects (Vonderohe 2009). Wisconsin Department of Transportation (WISDOT) has conducted research on adopting regulations for the application of AMC in highway construction projects. Their efforts are focused on the implementation strategies for mainstreaming 3D design models and DTMs in their projects. Duston and Montey (2009), in collaboration with Indiana Department of Transportation (INDOT), have also investigated the issues for streamlining the application of AMC systems.

\subsection{Administrative Infrastructure}

In order to take full advantage of AMC technology, it is important for project owners to develop specifications for the use of this technology, and require the contractors to follow those specifications. The specifications must cover: accuracy limits, quality assurance and quality control (QA/QC) procedures, risks allocation for errors, and payment mechanisms. Furthermore, there is a need to develop an implement ation strategy for adopting this technology in construction projects. Vonderode (2007) and Duston and Montey (2009) have reviewed the implemented measures and adopted practices of various Departments of Transportation (DOTs) in the US for accommodating AMC-friendly administrative infrastructure. These reports cover the types of AMC equipment allowed by DOTs and address the subsequent liability and financial issues. It could be construed from these reports that the terms AMC and AMG are used interchangeably. However, the reports provide evidences that suggest the DOTs are moving towards AMC, particularly with their numerous references to grading operations. As a result, to maintain the terminological consistency, we unified the terms to AMC. Table 1 summarizes the state-of-practice and administra- 
tive adjustments made towards streamlining the application of AMC in construction projects based on the above reports (Duston and Montey 2009, Vonderode 2007). It categorizes the DOTs' state of practice into used technologies, obligations of the contractor, documents and data format provided by DOTs, liability issues regarding error correction and data adjustment, incurred costs and QA/QC.

\subsection{Multi-agent Systems}

The concept of agents comes from developing thinking machines with the capability of solving problems (Shoham and Leyton-Brown 2009). Agents are relatively independent, selfish, and autonomous entities that operate within communities in accordance with complex modes of cooperation, conflict resolution, and competition in order to survive and perpetuate themselves (Russel and Noryig 2003). By exchanging information and coordinating with other agents, they can acquire more information about the environment and make better decisions that maximize their own utilities. There are several ways of planning for MASs either in a centralized or distributed manner (Durfee 1999). The centralized method treats the entire team as a single complex agent and then generates plans for this agent, whereas the distributed method generates plans for individual agents and uses coordination techniques to combine these plans. Each agent can generate a partial plan independently and the coordination of these partial plans can be centralized or distributed to form a single coherent overall plan (Shoham and Leyton-Brown 2009).

\subsection{Real-Time Simulation}

Simulation models provide the ability to capture the complexity of construction operations beyond simple mathematical formulation and has been used to analyze construction operations on a limited scale. Usually, those models are used at the planning phase to evaluate alternative construction scenarios (Lu, Dai and Chen 2007). A productivity improvement of $30 \%$ to $200 \%$ in the virtue of applying simulation is reported in the work of Halpin and Martinez (1999). Zayed and Halpin (2001) implemented Discrete-Event Simulation (DES) for the study of concrete batch-plant operations. Hassan and Guber (2008) approached investigation and optimization of Continuously Reinforced Concrete Pavement (CRCP) operations through computer simulation. Halpin and Martinez (1999) developed a Cyclone-based model to simulate the construction of a breakwaters and wharves using floating caissons.

However, traditional simulation models use statistical data to estimate task durations. In order to make the simulation results more realistic, real-time simulation has been suggested by several researchers. Song et al. (2008) have described a framework of real-time simulation for modeling heavy construction operations. Compared with traditional simulation, real-time simulation has the potential to improve the accuracy of performance forecasting while reducing modeling burdens on end users. Recent developments of RTS support decision making and control during project execution by overcoming some of these challenges (Hammad and Zhang 2011; Lu, Dai and Chen 2007).

Using RTS allows for examining different courses of action that a decision maker can take to correct the performance of a construction process and keep it on target in response to changes that happen on site.

\subsection{Path Planning}

Path planning is one of the major issues that need to be addressed in the development of multiple construction equipment control. A path planning algorithm computes a trajectory from the equipment present pose to a desired future pose. Kang and Miranda (2006) developed a kinematic and dynamic algorithm to generate efficient and smooth paths of cranes. However, they did not consider updating those paths in real time to avoid collisions.

In our previous research (Albahnassi and Hammad 2012; Zhang and Hammad 2012a; Zhang and Hammad 2012b; Zhang, Hammad and Bentahar 2011; Zhang, Hammad, and Rodriguez 2012) we developed several algorithms for path planning and re-planning for cranes. However, this previous research was limited to cranes and did not consider other types of construction equipment. 


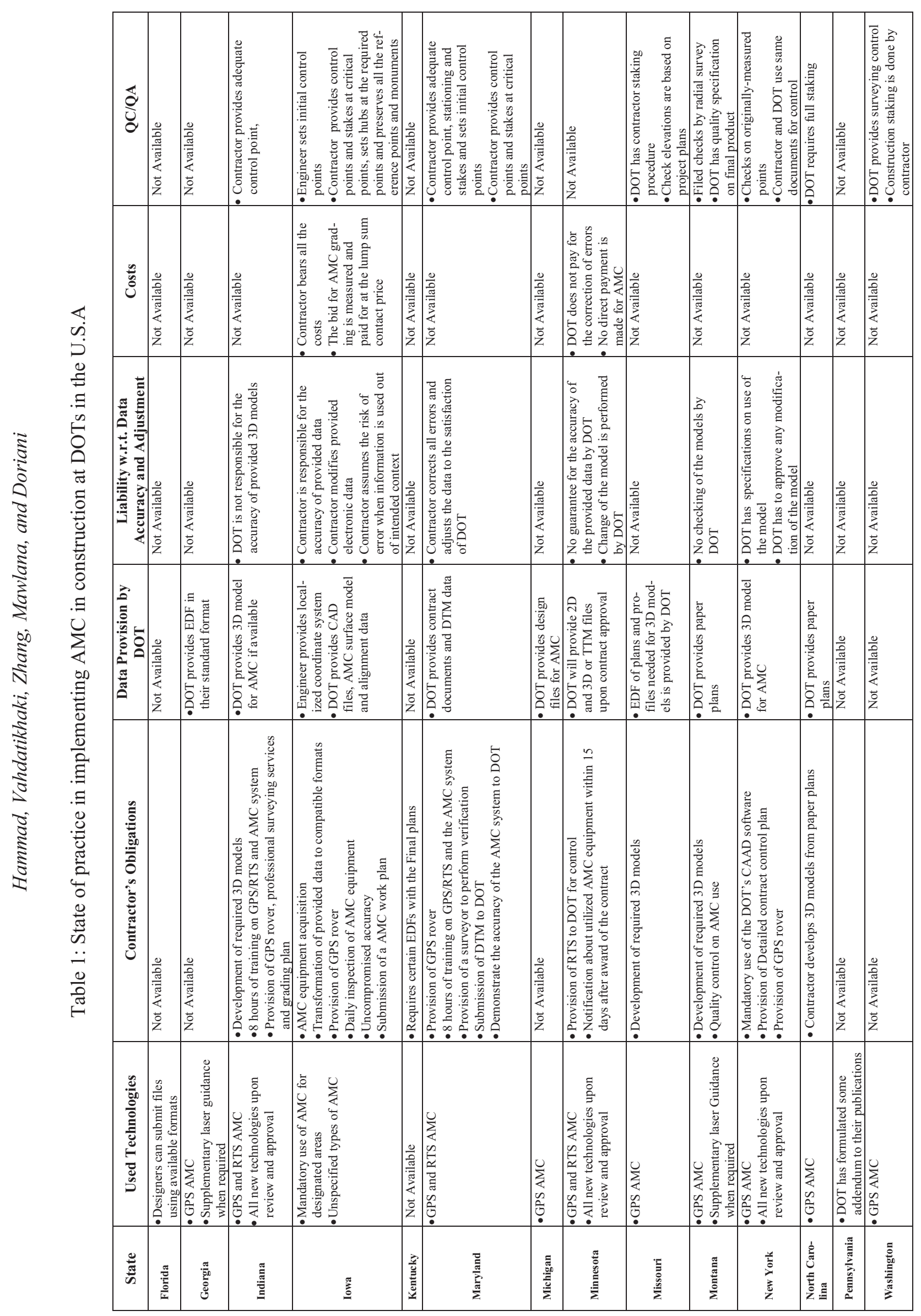




\section{PROPOSED APPROACH}

Our proposed approach is based on integrative application of multi-agent architecture and real-time simulation in AMC-equipped construction project settings for boosting the productivity and safety of construction operations. In the context of this approach, AMC is employed beyond the guidance/control of single on-site units, and transcends to an enabler for complex data handling and real-time analysis of operations. Accordingly, the assumption of this approach is that all construction machines are equipped with GPS so that their spatial position and relative orientation can be continuously tracked. The full-fledged implementation of the proposed method requires elaboration on methods used for RTS, and MAS communication and conflict resolution mechanisms. Similarly, it is required to defines how machine control decisions will be translated into machine-operable commands using appropriate path-planning algorithms.

\subsection{The Architecture of the Proposed Approach}

A multi-layer agent architecture is proposed in which agents representing single machines constitute the lowermost layer of the agent hierarchy. These agents process and manage the huge amount of collected sensory data into useful information that can be used in decision making at different operation and management levels. Figure 1 shows a simplified version of the proposed MAS architecture where several teams are working in proximity to each other. Four functional components could be distinguished according to the distribution of the responsibilities:

(1) Coordination: Coordination encompasses agents representing site coordinators. Within this component, two types of agents are distinguishable, namely the General Coordinator Agent (GCA) and Team Coordinator Agents (TCAs). The coordination agents will make critical decisions, e.g. new work plans or command for suspension of the operation, using data from all other components, and further communicate their decisions with appropriate OAs for execution. Essentially, this component consists of GCA and TCA, however depending on the characteristics of the project, the phase of the project and simultaneous operations, several teams and sub-teams can be formed. Each team is coordinated and supported by a TCA. The role of a TCA is to assign tasks to the subordinate OAs and sub-TCAs and to collect information from them. The TCAs also communicate the necessary re-planning decisions from and to the OAs and the GCA. Other example tasks of a TCA are: (a) Checking the equipment utilization and planning maintenance schedules at the team level; (b) Managing load counts, cycle times and utilization in order to optimize performance (e.g., estimated arrival times, travel times, and on-road times for permitting requirements); and (c) Giving safety warning to nearby agents when a potential collision is detected.

The GCA is responsible of monitoring and controlling the tasks at the project level to insure the smooth performance of the operations of all teams. For this purpose, the GCA interacts with real-time simulation component and communicates its results to the TCAs. Example tasks of the GCA are: (a) Analyzing the equipment utilization and productivity at the project level; and (b) Checking and controlling subcontractors' activities. Upon the detection of any organizational or operational conflicts, the MAS automatically generates an alternative work plan and informs all involved entities.

(2) Operations on site: the lowermost agent layer in the hierarchy of agents is OAs. These agents support the operators of each machine to accomplish their tasks. Example tasks of an OA are: (a) Providing a path to control a machine based on the DTM, the 3D design model, location of underground utilities, and the kinematics and engineering constraints of the machine; (b) collecting data about the work done by the machine and reporting to the respective TCA; (c) checking the equipment utilization; and (d) managing the compliance of operators and drivers with regulations (e.g., on road speeds).

(3) Information Sources: This component is in charge of handling the information required for machine control and encompasses Site State Agent (SSA) and Design Document Agent (DDA). The SSA provides information about the site (e.g. the DTM obtained by Light Detection and Ranging (LIDAR) scans), as the ingredient for the decision-making done by the coordinator agents and the forecasting per- 


\section{Hammad, Vahdatikhaki, Zhang, Mawlana, and Doriani}

formed by real-time simulation. The DDA furnishes the 3D design models and updates them should any change be made in the course of the project.

(4) Real Time Simulation: RTS is responsible for the calculation of the productivity and duration of forthcoming construction process using simulation methods based on real-time information gathered from the previous components. This responsibility includes, but is not limited to, forecast on the productivity of different operations, given the planned resource allocation scheme and work settings. Additionally, RTS, in integration with the object-oriented 3D models provided by the DDA, would contribute to detecting the time-space conflicts that may arise among concurrent tasks. The outcomes of the real-time simulation will be communicated with the coordinator agents for analysis and decision-making.

Our proposed approach is established on a hybrid of centralized and de-centralized MAS structure, meaning that a limited extent of autonomy is vested in the individual OAs and TCAs so that they can unilaterally take actions when due. This structure entails development of a communication protocol wherein the scope of agents' autonomy is defined and the framework for the flow of commands between different components is built. This protocol will define when and under which circumstances the agents can intervene, and through what mechanisms the changes they make are approved. It should be emphasized that the role of agents is to support the decision-making process and not to replace the human operators and coordinators. In this sense, agents will present the required information to the users given the users' position and the context of the information using different levels of detail. For instance, the digital display mounted in the cabin of the machine can show data about the path of the blade of the machine and about the sequence of activities the machine needs to follow. However, the display in the management office can illustrate the productivity curves of the machines, the maintenance/fuel requirement of machines, the delayed activities, etc.

\subsection{Real-time Simulation}

The simulation model will have to represent a real-world operation through interrelations of the time and resources associated with the independent time-consuming elements of that operation. Real-time simulation, though, will frequently update the initially set time/resource values of operation elements to better represent dynamism and volatility of the surrounding environment. In the proposed approach, data about the current as-built statues of the operation and site conditions will be provided via DDA and SSA respectively. Additionally, the present status of the equipment could be reflected via corresponding OAs, TCAs and/or GCA. Using this information, real-time simulation could more realistically perform operation forecasts in desired intervals or at critical times. It needs to be emphasized that the notion of real-time should not be construed as an uninterrupted chain of simulations as the operations proceed, but instead it should be understood as periodically performed simulation in intervals that is determined by the critically of the operations or when a simulation is requested by the GCA in response to an urgent unforeseen occurrence.

In this sense three roles could be defined for RTS in the proposed approach:(1) To report to the GCA (2) to provide updated forecast about the operations triggered in response to design change update reported by DDA; or (3) to simulate the various scenarios requested by GCA when an unforeseen circumstance befalls. The GCA will generate a set of possible scenarios through varying the time/resource values associated with the elements of an operation and request from RTS the feedback on performance, e.g. productivity and cost, of different scenarios. Subsequently, the RTS will present the results to GCA for selection of the optimum scenario.

\subsection{MAS Communication and Conflict Resolution}

The TCAs provide intelligent behavior for coordinating the work of OAs working together on the same task where their motion planning should be coordinated. The TCAs can also resolve conflicts among different teams of AMC machines working on different tasks in proximity of each other. The teams may belong to different contractors and may not be willing to compromise their own interests. In this case, the GCA is responsible for coordinating the work based on the project priorities. 
Once a potential conflict is detected, the involved TCAs communicate with the GCA by exchanging messages and they make decisions based on negotiation. For example, if re-planning is needed, the GCA decides which team has a higher priority. The priorities of the teams are decided according to several criteria such as the criticality of the task. If more than one priority rule are applicable, a conflict may occur between these priorities. The overall priority can be calculated based on functions that quantify the priorities with relative weights, and sum up these priorities as a single priority value.

\subsection{Path Planning and Real-Time Re-planning}

As explained in Section 2.5, robotic path planning and real-time re-planning algorithms have been applied to cranes. In the present approach, similar algorithms are under development for different construction equipment such as hydraulic shovels and backhoes. The algorithms provide real-time motion planning efficiently and safely in a complex environment with known and unknown obstacles while satisfying the engineering constraints of each machine. The motion planning algorithms utilize continuous collision detection queries to search the configuration space (Choset et al. 2005) for optimized feasible paths. The input data of the algorithms include construction equipment definition (kinematic properties, engineering constraints and geometrical representation) and environment definition (static and dynamic obstacles). The proposed algorithms are based on the Rapidly-exploring Random Trees (RRTs) which is a randomized sampling planning algorithm (LaValle 2006).

Applying RRT for re-planning in the case of a potential collision can be time-consuming since it abandons the initial tree and grows a new tree from scratch. For this reason, a re-planning algorithm based on Dynamic RRT (Ferguson 2006, Zhang and Hammad 2012b) is proposed for repairing the tree when necessary in a prioritized approach for efficient multi-equipment motion planning.

\subsection{Perceived Advantages of the Proposed Approach}

The benefits of the proposed approach will transcend those of the operation-level AMC implementation elaborated in Section 2.1. The proposed approach offers improvement in productivity beyond optimization of a single operation by considering the interdependencies of operations. In this context, safety issues and conflict-prone activities will be considered beyond single operations and the impact of modifications could be evaluated over the entire project. Moreover, our proposed approach will integrate the operational advantages of AMC with additional benefits at the managerial level by allowing managers to make informed decisions about the project using real-time data and simulation data.

\section{CASE STUDIES}

Given that the above proposed approach is still in the conceptual phase, there is no actual implementation of the approach in a real-world case study. However, to clarify the scope of the approach and to demonstrate various managerial and operational levels at which it could enhance decision-making, three hypothetical case studies are envisioned.

\subsection{First Case Study: Avoiding Collisions of Cranes}

The first case study is based on the operation of multiple cranes assigned to the installation of bridge segments in proximity of each other and demonstrates the operational level contribution of the proposed approach. The OA of the crane has the knowledgebase that includes the kinematic constraints, the engineering constraints, and the rules for actions of the crane. Taking hydraulic cranes as an example, the kinematic constraints, i.e., the degrees of freedom, can be defined according to the specifications. Engineering constraints are based on the working range and load charts. The working range shows the minimum and maximum boom angle according to the length of the boom and the counterweight. Load charts give the lifting capacity based on the boom length, the boom angle to the ground, and the counterweight. 


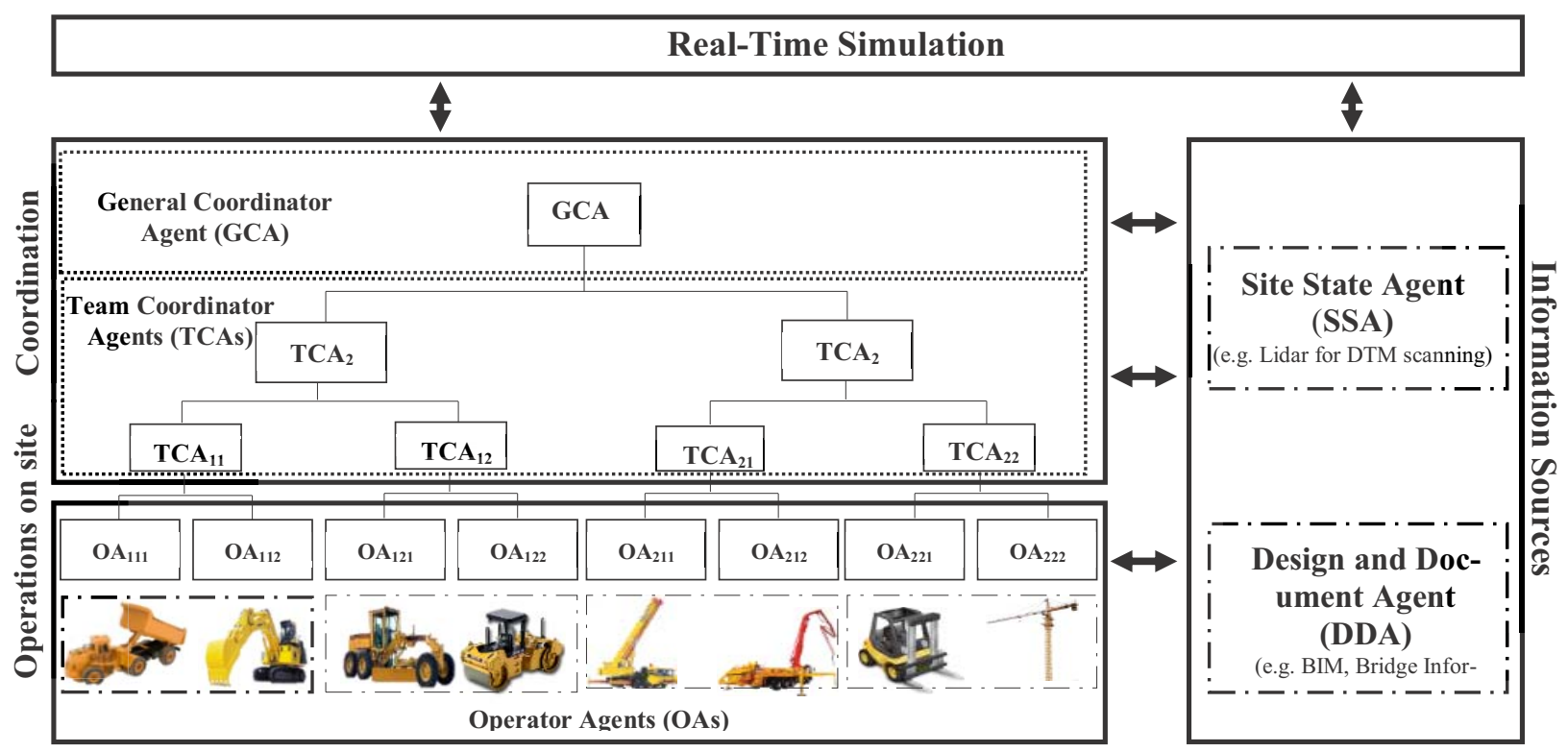

Figure 1: Simplified MAS Architecture

Ultra-Wideband (UWB) technology is used to track the movement of the cranes (Hammad and Zhang 2011). UWB tags are attached to different components of the cranes to monitor the poses (i.e., the position and orientation) of those components. These poses are used by the OAs to detect potential collisions with obstacles on the path and to ensure safety (Zhang, Hammad, and Rodriguez, 2012). Using the location data of the moving objects on site, the $O A$ applies collision detection for the next movement. If it is not collision-free, re-planning is triggered and a new motion path is generated (Zhang and Hammad, 2012b). There are several TCAs managing different areas on site. Once a potential conflict is detected, the involved $O A$ communicates with the $T C A$ to report the situation. If re-planning is needed, the $G C A$ decides which crane has higher priority. The agent of the higher-priority crane resumes its path and the other agent re-plans the path of the lower-priority crane. The priorities of the OAs are decided in terms of safety, time, and cost, in order to select which agent should re-plan the path (Zhang and Hammad, 2012a).

As shown in Figure 2, it is assumed that the task of the crane on the left hand side is on the critical path of the project; consequently, the TCA gives the priority to that crane to guarantee the project is not delayed (Figure 2(a)). If a potential collision was detected (Figure 2(b)), the low-priority crane (on the right hand side) will re-plan its path to avoid being obstacle of the high-priority crane (Figure 2(c)).

\subsection{Second Case Study: Avoiding Underground Utilities}

The second case study aims to depict the operational level scenario in which the proposed approach helps the project managers effectively respond to an unforeseen accident. In this case study, a fleet of earthmoving machines are working on an embankment operation. It is conceivable that an undetected underground utility runs beneath the area where excavators are cutting the ground. As shown in Figure 3, the backhoe on the left has uncovered an underground pipe while excavating. The collision with this utility, depending on the criticality and the type of the utility, could result in tremendous delays and safety threats. In the context of the proposed approach, however, it is assumed that the excavator is equipped with AMC and also represented by a semi-autonomous agent.

For the excavator to be able to proceed either with autonomous decision-making or communication with the responsible TCA for complementary prescriptions, an underground utility detection mechanism is needed. This mechanism could be within a range of available solutions from conventional buried warning tapes to state-of-the-art sensory technologies, e.g. Radio Frequency Identification (RFID). When the excavator OA detects that a collision with underground utility could occur, it autonomously stops the ex- 
cavator. Simultaneously, a notification is sent to the responsible TCA, which will communicate the message to the GCA and all affected TCAs. Consequently, the GCA will have a range of solutions to select from. If the detected underground utility does not directly disrupt the project, the GCA will dispatch caution alarm to all affected OAs and require them to move carefully around the detected utility.

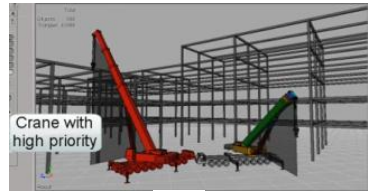

(a)

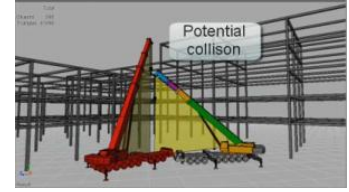

(b)

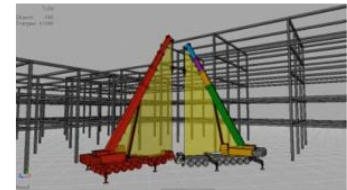

(c)

Figure 2: Coordinating the operations of two cranes

However, if the underground utility is disrupting the continuation of the project, the main two options are to make a design change request to the office to reroute the road segment or to relocate the underground utility. Meanwhile, the GCA will re-assign the affected machines to a different task and will communicate that decision to the relevant TCAs. In each of the above cases, the alternative scenarios will be simulated to predict the most productive case.

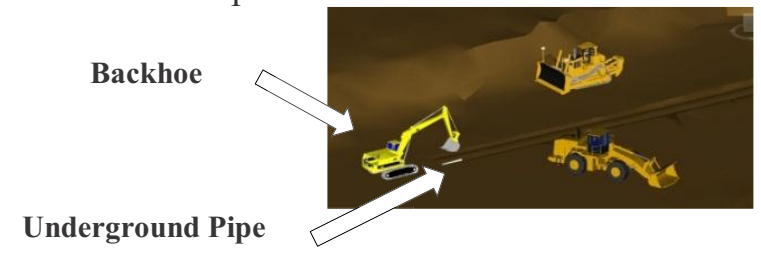

Figure 3. Re-planning to avoid accidents related to underground utilities during excavation operation

\subsection{Third Case Study: Avoiding Scheduling Conflicts}

This case study will be in the context of the Turcot Interchange project in Montreal, Canada, and mainly focuses on the managerial level contribution of GCA and TCAs. The existing interchange is composed of several bridge structures located at three different levels, with a maximum height of 28 meters and an average height of 18 meters. In projects of this scale, scheduling spatio-temporal conflicts may occur if the project execution plan was not done or followed effectively (Hammad, Mawlana and Doriani 2012). In this case study, it is assumed that one subcontractor is working on the subproject of constructing segment $\mathrm{G}$ of the new interchange, while another subcontractor is responsible for the demolition of segment $\mathrm{F}$ of the existing interchange as shown in Figure 4. If a delay occurs in the demolition of segment F, the TCAs involved in that segment will detect, based on earned value or progress reports, this delay and report it to the GCA. Real-time simulation will be then performed to examine the impact of this delay on the project execution plan. The results from the real-time simulation will be integrated with the DDA to perform spatio-temporal analysis. If a spatio-temporal conflict exists, it will delay the progress of the segment under construction which in return may delay the project and cause an overrun in the budget. Figures 4 (a) and (b) represent the results of discrete event simulation combined with a 3D model of the interchange. As shown in Figure 4(a), the initial plan of project execution will not cause any spatio-temporal conflicts between the segment under construction and the one under demolition. On the other hand, a delay in the demolition of segment $\mathrm{F}$ in the execution phase (Figure 4(b)) will result in a spatio-temporal conflict. The GCA will investigate a range of modification of assigned resources and suggest several scenarios on how to deal with this conflict. Each scenario will be evaluated using simulation. Next, the MAS will review the feasible scenarios and select the scenario that has the least impact on the project objectives. Finally, appropriate commands will be generated and dispatched to the TCAs and OAs for implementation. 


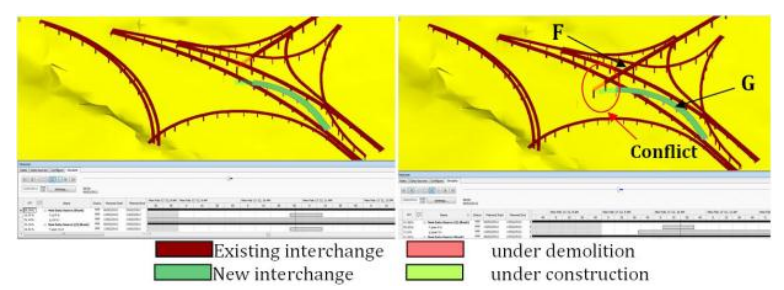

(a) Planning Phase (b) Execution Phase

Figure 4. Snapshots of the developed 4D models of the existing and new interchanges

\section{CONCLUSIONS AND FUTURE WORK}

The presented study introduced a novel approach for improving the productivity and safety of construction projects, exploiting the advantages of multi-agent systems architecture, real-time simulation and automated machine control. In this approach a multi-layer agent-based architecture is designed to monitor, coordinate and control the operations of construction machines and to resolve managerial conflicts. The proposed MAS structure is underpinned by four distinct components, namely coordination, operation on site, information sources and real-time simulation. This structure would allow to organize construction machines into teams and establish two-way communication and command channels between operationlevel agents and coordination-level agents. The system is continuously endorsed by the information sources component which furnishes the information needed for decision-making and analysis. Real-time simulation is used to empower the decision-making with continuous analysis of productivity and safety. Several hypothetical case studies were developed to demonstrate the breadth of the solutions this approach could provide and the different levels at which it could be effective.

In the light of the findings of this paper it could be concluded that: (1) Implementation of such a broad approach requires more than the study of the technical aspects and it is required to identify the administrative infrastructure required to formalize the applications of AMC in construction; (2) The proposed MAS structure has potentials to immediately preempt delays or safety threats by rapidly generating several alternative scenarios and performing analysis for the most effective solution; and (3) A wide variety of operational and managerial conflicts could be effectively addressed using the proposed approach.

In order to have a full-fledged implementation of this approach, our future work will include : (1) Administrative infrastructure: it is required to develop questionnaires to collect data about the state-ofpractice in transportation agencies in Canada and the U.S. and recommend the prerequisite measures needed to accommodate AMC more effectively. It behooves us to use Unified Modeling Language (UML) (Martin and Scott 2000) to formalize the interaction between contractors and transportation agencies and clarify the most efficient workflow; (2) MAS algorithm: the proposed MAS structure needs to be developed and implemented; (3) Real-time simulation: It is necessary to identify the most appropriate simulation technique and further bridge the simulation engine to a 3D model for the purpose of 4D visualization and clash detection; (4) MAS conflict resolution: the algorithms and methods to resolve issues arising from conflict of interests between several agents needs to be addressed and pattern s for negotiation, collaboration and communication of the agents needs to be developed; (5) Path-planning and machine-control: the translation of the MAS-made decision into machine-operable motions needs to be scrutinized; and finally, (6) The integration of the above studies needs to be implemented in actual case studies to validate the expected outcomes.

\section{ACKNOWLEDGMENTS}

The authors would like to extend their thanks to Drs. Siman Abourizk, Jamal Bentahar, Khashayar Khorasani, and Yasser Mohammad for their feedback on an earlier version of this paper submitted as a part of a research proposal. Also, the authors would like to thank Mr. Shayan Setayeshgar, graduate student and research assistant at Concordia university, who helped in the development of the 3D and 4D models. 
Hammad, Vahdatikhaki, Zhang, Mawlana, and Doriani

\section{REFERENCES}

Albahnassi, H., and A. Hammad. 2012. "Near real-time motion planning and simulation of cranes in construction: Framework and system architecture", Journal of Computing in Civil Engineering. 26(54).

Beavers, J.E., J. R. Moore, R. Rinehart, and W. R. Schriver. 2006. "Crane-related fatalities in the construction industry." Journal of Construction Engineering and Management, ASCE, 132(9):901-910.

Choset, H., K.M. Lynch, S. Hutchinson, G. Kantor, W. Burgard, L.E. Kavraki, and S. Thrun. 2005. "Principles of Robot Motion - Theory, Algorithms, and Implementations", The MIT Press, Cambridge.

Dunston, P.S., and J. Monty. 2009. "Practices for seamless transmission of design data from design phase to construction equipment operation - a synthesis study", Purdue University, West Lafayette, Indiana.

Durfee, E. 1999. Distributed problem solving and planning, Multiagent Systems: A Modern Approach to Distributed Artificial Intelligence. Gerhard Weiss (ed.). MIT Press.

Ferguson, D. 2006. "Single agent and multi-agent path planning in unknown and dynamic environments." $\mathrm{Ph} . \mathrm{D}$. dissertation of the Robotics Institute, Carnegie Mellon University, Pennsylvania.

Frantzen, C., S. Kirn, M. Kaak, A. Sturm. 2010. "Research project AutoBauLog - Automatic control in construction logistics.", Proceeding of the 2nd International Conference on Machine Control and Guidance, Bonn.

Halpin , D. W., and L. H. Martinez. 1999. "Real World Applications of Construction Process Simulation." Proceeding of the 1999 Winter Simulation Conference.

Hammad, A., M. Mawlana, A. Doriani. 2012. "Discrete event simulation and 4D modeling for elevated highway reconstruction projects", Proceeding of the 14th International Conference on Computer in Civil and Building Engineering, Moscow.

Hammad, A., and Zhang, C. (2011). Towards real-time simulation of construction activities considering spatio-temporal resolution requirements for improving safety and productivity, Winter Simulation Conference, IEEE, Phoenix.

Hassan, M. M., and S. Gruber. 2008. "Simulation of Concrete Paving Operations on Interstate-74." Journal of Construction Engineering and Management

Jamasmie, C. 2010. "The mine of the future might be a thing of the past." MINING.com. pp. 6-9.

JCMMRI. 2012. Japan Construction Methods and Machinery Research Institute, http://www.jcmanet.or.jp/english/cmi.html.

Kang, S., and E. Miranda. 2006. "Planning and visualization for automated robotic crane erection processes in construction", Automation in Construction, 15:398-414.

LaValle, S.M. 2006. Planning Algorithms, Cambridge University Press, New York.

Lu, M., F. Dai, and W. Chen. 2007. "Real-time decision support for planning concrete plant operations enabled by integrating vehicle tracking technology, simulation, and optimization.", Canadian Journal of Civil Engineering 34:912-922.

Martin, F. and K. Scott. 2000. "UML Distilled (2nd Ed.): A Brief Guide to the Standard Object Modeling Language.” Addison-Wesley Longman Publishing Co., Inc., Boston, MA, USA.

Noland, R. 2010. "Site connectivity - Positioning \& machine control's next technology step, Machine Control Online." http://machinecontrolonline.com/content/view/6390/136/

Perkinson, C. L., M. E. Bayraktar, and I. Ahmad. 2010. "The use of computing technology in highway construction as a total jobsite management tool." Automation in Construction, 884-897.

Peyret, F., J. Jurasz, A. Carrel, E. Zekri, and B. Gorham. 2000. "The computer integrated road construction project." Automation in Construction, 9:447-461.

Russell, S., and P. Norvig. 2003. Artificial Intelligence, A Modern Approach, Prentice Hall.

Rausch, P., F. Schreiber, and M. Diegelmann. 2010. "Close loop cotrolling approaches for projects in the earth moving and road construction industry." Proceeding of the 2nd International Conference on Machine Control and Guidance, Bonn.

Shoham, Y., and K. Leyton-Brown. 2009. Multiagent Systems. Algorithmic, Game-Theoretic, and Logical Foundations. Cambridge University Press. 
Song, L., F. Ramos, and K. Arnold, 2008. "A framework for real-time simulation of heavy construction operations." In the Proceeding of the 2008 Winter Simulation Conference, Edited by S.J. Mason, R.R. Hill, L. Monch, O. Rose, T. Jefferson, J. W. Fowler, 2387-2395. Piscataway, New Jersey.

Teizer, J., B. S. Allread, C. E. Fullerton, and J. Hinze. 2010. "Autonomous pro-active real-time construction worker and equipment operator proximity safety alert system." Automation in Construction, 630-640.

Torres, H.N., and M. Ruiz. 2011. "Improving Highway Project Delivery." TRB Annual Meeting, Washington DC.

Vonderohe, A.P. 2007. "Implementaion of GPS controlled Highway Construction Equipment." Construction Materials and Support Center, University of Wisconsin.

Vonderohe, A.P. 2009. "Report of status and plans for implementing technologies for design and construction in WisDOT." Construction Materials and Support Center, University of Wisconsin.

Zayed, M. T., and D. Halpin. 2001. "Simulation of Concrete Batch Plant Production." Journal of Construction Engineering and Management.

Zhang, C., and A. Hammad. 2012a. "Multi-agent approach for real-time collision avoidance and path replanning on construction sites". Journal of Computing in Civil Engineering, ASCE (Available online).

Zhang, C., and A. Hammad. 2012b. "Improving lifting motion planning and re-planning of cranes with consideration for safety and efficiency". Journal of Advance Engineering Informatics (Available online).

Zhang, C., A. Hammad, and H. Albahnassi. 2009. "Collaborative multi-agent systems for construction equipment based on real-time field data capturing." ITcon, Vol. 14: 204-228.

Zhang, C., A. Hammad, and J. Bentahar. 2011. "Multi-agent-based approach for real-time collision avoidance and path re-planning on construction sites." 28th Int. Symp. on Autom. \& Robotics in Const., Seoul.

Zhang, C., A. Hammad, and S. Rodriguez. 2012. "Crane pose estimation using UWB real-time location system". Journal of Computing in Civil Engineering, ASCE (in print).

\section{AUTHOR BIOGRAPHIES}

AMIN HAMMAD is a Professor at the Concordia Institute for Information Systems Engineering. His research focuses on sustainable infrastructure lifecycle management systems and investigates several methods and techniques including spatio-temporal information modeling and analysis, simulation, visualization, optimization, wireless communications, sensing, auto-identification, and real-time location tracking. His email is hammad@ciise.concordia.ca.

Faridaddin Vahdatikhaki is a $\mathrm{PhD}$ student in Building, Environmental and Civil Engineering Department (BCEE) at Concordia University. His research interests are automation in construction, simulation in construction, and Knowledge, management systems. His email address is f_vahdat@encs.concordia.ca.

Cheng Zhang is a Post-Doctoral Follow at Concordia Institute for Information Systems Engineering. Her research interests are in automation in construction, improving safety on construction site, and real-time location systems. Her email address is 1ilyzc2003@hotmail.com.

Mohammed Mawlana is a Research Assistant and PhD student in BCEE at Concordia University. His research interests are automation in construction and simulation optimization. His e-mail is m_mawlan@encs.concordia.ca

Ahmad Doriani is graduate student in BCEE at Concordia University. His research focuses on highway reconstruction planning and scheduling using Building Information Modeling (BIM) techniques, automation in construction. His email address is a doria@encs.concordia.ca. 